\title{
Cortical Blindness In Fat Embolism Syndrome Following Fracture Manipulative Procedure
}

\author{
A Shahrulazua, MMed Ortho (USM), MS Ariff*, MS Ortho (UKM), MA Aziz**, MS Ortho (UKM) \\ Department of Orthopaedics and Traumatology, Universiti Kebangsaan Malaysia Medical Centre, Kuala Lumpur, Malaysia \\ *Orthopaedic unit, Shah Alam Specialist Hospital, Shah Alam, Malaysia \\ ${ }^{* *}$ Orthopaedic unit, An-Nur Specialist Hospital, Bangi, Malaysia
}

\begin{abstract}
Fat embolism syndrome is a well-recognised sequela of long bone trauma as well as intramedullary orthopaedic procedures. However, it has rarely been described following manipulation, reduction of fracture, and application of external fixator. Furthermore, bilateral ocular blindness is seldom the first manifestation; instead respiratory and other cerebral symptoms being most common. We describe a case with this rare presentation in a patient who underwent a trial of closed reduction, then open reduction of a femur fracture, followed by external fixation performed at day 47 post-initial trauma.
\end{abstract}

\section{Key Words:}

Cortical Blindness, Visual Blindness, Fat Embolism, Fracture Manipulation, Fracture Reduction

\section{INTRODUCTION}

Fat embolism syndrome frequently presents within 72 hours after the initial insult. We report a provocative case of cerebral failure in which initial presentation was bilateral blindness followed by lung dysfunction; this occurred following manipulation, reduction and external fixation of femur fracture.

\section{CASE REPORT}

A physically fit 30-year-old man was involved in a motorvehicle accident and sustained multiple fractures to his right lower limb, consisting of an open Grade 3A fracture of the distal third femur, a tibial plateau fracture, as well as an open patella fracture (Figure 1). He underwent emergency wound debridement and calcaneal skeletal traction was applied before transferred to our tertiary centre. Since, upon admission to our centre, subcutaneous wound infection was noted over his right knee, an open reduction and 'cross-knee' external fixation was performed to treat the femur and tibia fractures, and screw fixation was used for the tibial plateau fracture. The patient was subsequently discharged on wheelchair mobilisation and was not bed-ridden whilst at home.
Unfortunately, the patient had a fall at home that led to diagnosis of further displacement of his femur fracture during a follow-up appointment on the 43rd day following the motor-vehicle accident (Figure 2). On the 47 th day posttrauma, a second open reduction and application of a new external fixator was performed following a failed attempt at revision of the external fixator and close reduction. The surgery was uneventful and the estimated blood loss was only $200 \mathrm{mls}$. Approximately 24 hours following the surgery, the patient complained of feeling tired and was noted to have a temperature of $39^{\circ} \mathrm{C}$. He slept after he was given paracetamol, but woke up approximately 5 hours later with a severe headache, bilateral blindness, nausea and vomiting. He denied any chest pain or shortness of breath, Glasgow Coma Scale was normal, blood pressure was $66 / 44 \mathrm{mmHg}$, and pulse was 120 beats per minute. Respiratory examination was normal. No focal neurological abnormalities were detected, but visual acuity was zero, yet fundoscopy showed no obvious abnormality.

His central venous pressure was normal and arterial blood gases on room air were initially unremarkable with an oxygen saturation of $96.7 \%$. Haemoglobin decreased from $10.8 \mathrm{~g} / \mathrm{dl}$ preoperatively to $9.2 \mathrm{~g} / \mathrm{dl}$, and platelet count was reduced from $503 \times 109 / 1$ to $148 \times 109 / 1$. His blood biochemistry, coagulation profile and D-dimer screen were within normal levels. Electrocardiograph (ECG) showed only sinus tachycardia. He was initially resuscitated with intravenous fluid. As his blood pressure remained unstable, treatment with dobutamine and renal dose of dopamine was undertaken.

An urgent CT brain, performed on the same day, was reported as normal. After about 12 hours following the onset of bilateral blindness, he developed respiratory failure with deteriorating mental status. His chest radiograph showed diffuse bilateral perihilar infiltrates (Figure 3). Subsequent eye examination revealed bilateral fixed dilated pupils with persistent vertical nystagmus. A diagnosis of posterior cerebral artery circulation infarct was made, and he was then intubated and mechanically ventilated.

Corresponding Author: Shahrulazua Ahmad, Department of Orthopaedics and Traumatology, Universiti Kebangsaan Malaysia Medical Centre, Jalan Yaacob Latif, Bandar Tun Razak, 56000, Cheras, Selangor, Malaysia Email: drshahahmad@hotmail.com 

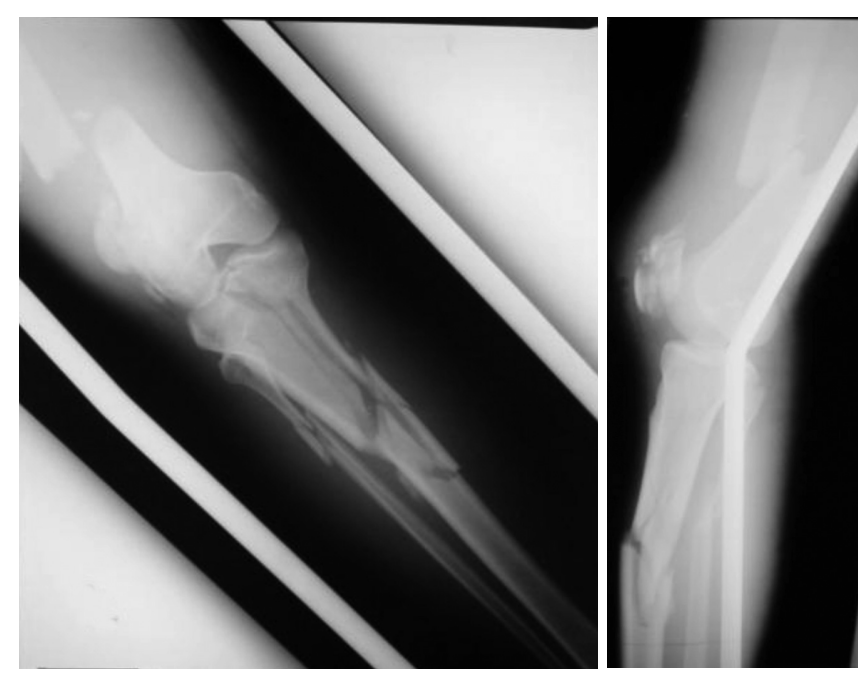

Fig. 1: Radiographs showing multiple fractures sustained at the right lower limb.

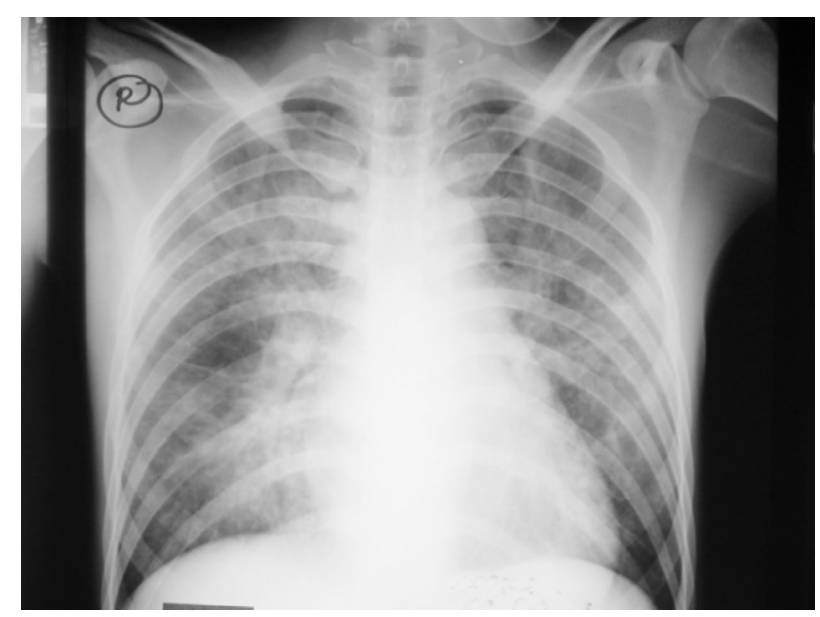

Fig. 3: Initial chest radiograph taken approximately 12 hours following the onset of bilateral blindness, showing diffuse bilateral pulmonary infiltrates.

On the third post-operative day, he was noted to have bilateral ischaemia of hands and feet, bilateral subconjunctival haemorrhages, and petechial rashes on his upper trunk. A repeat chest radiograph showed deterioration of his bilateral pulmonary congestion, which was worse on the right lung (Figure 4). Portable transthoracic echocardiograph showed an ejection fraction of $67 \%$ with no other significant finding apart from a dilated left atrium. Unfortunately, despite ventilatory and inotropic support, the patient succumbed on the fourth post-operative day from cardiopulmonary failure.

\section{DISCUSSION}

The diagnosis of fat embolism syndrome in this patient was made on the basis of the close temporal association between

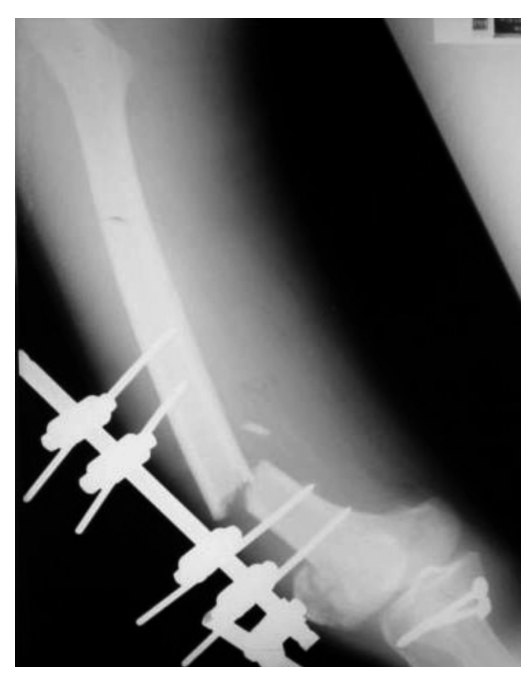

Fig. 2: Radiograph showing displacement of the femoral fracture fixation following a fall at home.

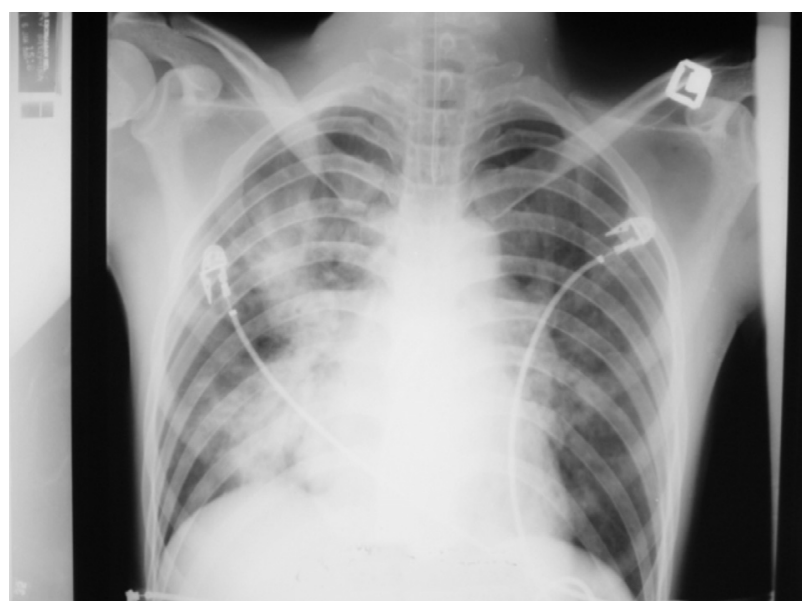

Fig. 4: Repeat chest radiograph taken on the third postoperative day showing increased bilateral pulmonary congestion, which was worse on the right lung.

the second surgery and the occurrence of his symptoms and signs. According to Gurd's criteria, clinical diagnosis of fat embolism syndrome requires at least one major feature plus four minor features ${ }^{1}$. In this case, the patient showed three major features consisting of central nervous system disturbance, respiratory insufficiency, and petechiae, as well as four minor features (pyrexia, tachycardia, thrombocytopenia, and anaemia) that were not logical sequela of the intraoperative blood loss.

The other possible differential diagnosis in this patient would have been a pulmonary embolism. The high sensitivity and negative predictive values potentially made a $\mathrm{D}$-dimer test an ideal test to exclude the presence of pulmonary embolism ${ }^{2}$. The D-dimer test was within normal range for this patient, thus it is highly unlikely that he suffered from a pulmonary embolism. As in this present case, most patients with fat 
embolism have a normal ECG, in contrast to those with pulmonary embolism that usually present with ST segment and T wave ECG abnormalities ${ }^{1,2}$.

Echocardiography is also considered to be valuable in differentiating shock states and recognising the characteristic features of pulmonary embolism ${ }^{2}$. Nonetheless, the portable transthoracic echocardiograph performed on this patient failed to show any abnormal masses or echogenic material within the cardiac chambers that would be the likely sources of pulmonary emboli. There was also no abnormal echocardiographic findings of right ventricular pressure overload often manifested in pulmonary embolism ${ }^{2}$. Furthermore, this patient was not bed-ridden after he was discharged from his first hospital admission, and there was no calf swelling or tenderness noted during his second admission to suggest the presence of deep venous thrombosis that could lead to pulmonary embolism.

The transthoracic echocardiograph performed showed that the ejection fraction was $67 \%$, a result possibly due to the effects of the inotropes that were administered to the patient. Cardiac stimulants such as dobutamine and dopamine mainly act on beta adrenergic receptors in cardiac muscle, and increase contractility with little effect on the rate. Hence, it was highly unlikely that these inotropic sympathomimetics had led to peripheral vasoconstriction or the ischaemic pathology.

A literature search yielded no previous report of fatal fat embolism syndrome following external fixation. In fact, this syndrome is more commonly associated with intramedullary orthopaedic procedures and those associated with methylmethacrylate cement application ${ }^{1}$. However in this case, we suspect that the trial of close reduction and the second open reduction procedure might have disrupted the femoral bone marrow causing the syndrome. This case further reaffirms the findings of a previous study, which showed that the levels of embolised fat does not seem to be affected by the different methods of fixation ${ }^{3}$.

This case is remarkable because of the unusual presentation of bilateral blindness as the early manifestation of the illness without any initial respiratory involvement. We are aware of only a few cases of retinopathic visual loss secondary from fat embolism of ocular vessels reported in the past ${ }^{4}$. In contrast, this patient failed to show any fundoscopic abnormalities. The bilateral blindness was likely cortical in origin although the brain CT brain failed to show any abnormalities. Brain CTs however are not the most sensitive test especially as compared to MRI, which has been reported to be the best tool for detection of brain infarcts in fat embolism syndrome ${ }^{1}$. Cerebral microembolism can also be diagnosed and monitored in real time using transcranial Doppler $^{5}$. In the present case, the embolism most likely involved the area of the posterior cerebral arteries resulting in cortical blindness as the presenting symptom.

In conclusion, fat embolism syndrome may present with acute bilateral blindness without significant fundoscopic or CT brain findings. Although rare, it may occur following manipulative procedures and external fixation of bone fracture. 


\section{REFERENCES}

1. Papagelopoulos PJ, Apostolou CD, Karachalios TS, Themistocleous GS, Giannakopoulos CK, Ioannidis TT. Pulmonary fat embolism after total hip and total knee arthroplasty. Orthopedics, 2003; 26(5): 523-7.

2. Wood KE. Major pulmonary embolism: Review of a pathophysiologic approach to the golden hour of hemodynamically significant pulmonary embolism. Chest. 2002; 121(3): 877-905.

3. Schemitsch EH, Jain R, Turchin DC, Mullen JB, Byrick RJ, Anderson GI, et al. Pulmonary effects of fixation of a fracture with a plate compared with intramedullary nailing. A canine model of fat embolism and fracture fixation. J Bone Joint Surg Am, 1997; 79(7): 984-96.

4. Chuang EL, Miller FS, Kalina RE. Retinal lesions following long bone fractures. Ophthalmology. 1985; 92(3): 370-4.

5. Forteza AM, Koch S, Romano JG, Zych G, Bustillo IC, Duncan RC, et al. Transcranial doppler detection of fat emboli. Stroke. 1999; 30(12): 2687-91. 